\title{
La producción editorial didáctica de la Universidad Estatal a Distancia de Costa Rica: un diagnóstico a partir del modelo de industrias de contenidos
}

Jenny Teresita Guerra González *

Artículo recibido: 5 de junio de 2014. Artículo aceptado: 9 de mayo de 2015.

\section{Resumen}

En este artículo se analiza la producción editorial didáctica de la Universidad Estatal a Distancia de Costa Rica (UNED), institución pública que imparte enseñanza a nivel superior en la modalidad a distancia. Se estudia al Programa de Producción de Material Didáctico Escrito (PROMADE) a partir de una metodología basada en el modelo de industria de contenidos para la convergencia digital. Las industrias de contenidos comprenden toda la producción pensada para las nuevas tecnologías de la información y comunicación, la convergencia digital y plantean la inclusión social. Incluyen la interactividad y movilidad posibilitadas por celulares, computadoras portátiles, tablets, entre otros dispositivos electrónicos.

* InstitutodelnvestigacionesBibliotecológicasydelalnformacióndelaUNAM, México.jenn_guerra@hotmail.com

INVESTIGACIÓN BIBLIOTECOLÓGICA,Vol.30,Núm.68,enero/abril,2016,México,ISSN: 0187-358X. pp. 125-153 
Palabras clave: Producción didáctica; Edición universitaria; Industrias de contenidos; Costa Rica.

\begin{abstract}
Publishing output in the field of didactics of the Universidad Estatal a Distancia de Costa Rica: A diagnosis on the basis of a model of industry content Jenny Teresita Guerra-González
\end{abstract}

This paper analyzes the publishing output in the field of education at the Universidad Estatal a Distancia de Costa Rica (UNED), a public, higher education, distance-learning institution. Researchers examined the Written Didactic Materials Production Program (PROMADE by its Spanish-language acronym) using a methodology based on a digital convergence model of the content industry. Content industries include all the output targeted for new information and communications technologies and digital convergence for the purpose of enhancing social inclusion. They also include the interactivity and mobility made possible by mobile phones, portable computers, tablets and other electronic devices.

Keywords: Publishing educational, university publishing, content industries, Costa Rica

\title{
El caso de estudio
}

a Universidad Estatal a Distancia de Costa Rica (UNED) fue creada en 1977 y toma como base los modelos educativos de la UNED de España y la Open University de Gran Bretaña, que buscaban atender a poblaciones dispersas, independientemente de su ubicación geográfica y de su disponibilidad temporal.

En el modelo de educación a distancia de la UNED interactúan tres variables: los estudiantes, el docente y los contenidos o el conocimiento. El estudiante es el actor y componente principal; la docencia desdobla la figura individual del docente tradicional en un conjunto de funciones que llevan a cabo varias personas, como los editores académicos y los autores de las Unidades Didácticas Modulares; y los contenidos mediatizan la relación entre 
el estudiante y el conocimiento, razón por la cual destaca la forma en que se ponen en contacto con el estudiante (Modelo Pedagógico..., 2007: 28).

La UNED cuenta con cuatro escuelas (Ciencias Exactas y Naturales, Ciencias de la Administración, Ciencias de la Educación, y Ciencias Sociales y Humanidades), el Sistema de Estudios de Posgrado y 45 centros universitarios distribuidos por todo el país, que son responsables de las tareas docentes. En la metodología a distancia, los centros universitarios son el punto de reunión del estudiante, el lugar donde realiza trámites administrativos y hace uso de diferentes servicios estudiantiles.

La práctica docente en esta institución se orienta a facilitar la búsqueda, el procesamiento y la asimilación de nuevo conocimiento por parte del estudiante. Según los planteamientos de la educación a distancia actual, la interrelación estudiante-"docente" se caracteriza por las transformaciones que se describen a continuación.

a) Un cambio del esquema "un profesor-muchos estudiantes" a un esquema "un estudiante-muchos docentes", entendidos éstos como fuentes de conocimiento y orientación diversos y dispersos.

b) Una intensificación del trabajo interdisciplinario y de equipo en la función docente a distancia, que comprende la planificación académica, el diseño y la producción de Unidades Didácticas Modulares (UDM), la facilitación del proceso de aprendizaje, así como la evaluación y autorregulación de los aprendizajes.

c) La coordinación efectiva entre los actores responsables de las diferentes acciones relacionadas con el diseño y producción de las UDM, apoyados en un concurso sobre los principios epistemológicos y pedagógicos expertos.

La Unidad Didáctica Modular (UDM) definida como "el material impreso básico que desarrolla los contenidos fundamentales de cada asignatura y cuyos elementos didácticos están orientados para que el alumno aprenda a aprender" (Lobo Solera y Fallas Araya, 2008: 63. Cursivas desde el original.) fue el parteaguas para la creación primero de una Oficina de Publicaciones en la UNED (mayo de 1977) y posteriormente, el 31 de enero de 1979, de la Editorial de la Universidad Nacional Estatal a Distancia de Costa Rica (EUNED), orientada a la publicación de textos de línea editorial. Durante 35 años la EUNED ha administrado la línea editorial de la institución a la cual pertenece y simultáneamente gestionado su sello (marca); es además una de las cuatro editoriales universitarias en este país que pertenecen a universidades públicas. 


\section{La producción de materiales didácticos}

La producción de materiales didácticos en la UNED es rastreable hasta agosto de 1977 cuando dio inicio la edición de libros de texto de los cinco cursos del Ciclo Básico. De esta primera fase (agosto 1977-febrero 1978) surgieron documentos que regularon el proceso editorial en la universidad por más de dos décadas, como el Contrato de Elaboración de Unidades Didácticas, el Reglamento de Selección de Autores yel Instructivo Metodológico, primerdocumento guía para autores de la institución.

El 1 de agosto de 1979 se creó la Dirección de Producción Académica (DPA), hoy Dirección de Producción de Materiales Didácticos (DPMD). La DPMD es una dependencia perteneciente a la Vicerrectoría Académica, instancia encargada de planificar, ejecutar, articular y evaluar con pertinencia y calidad los quehaceres relacionados con el proceso de enseñanza y aprendizaje en la UNED. ${ }^{1}$

La DPMD agrupa a la fecha cinco programas: Programa de Producción de Material Didáctico Escrito (PROMADE); Programa de Producción de Material Audiovisual; Programa de Producción Electrónica Multimedial; Programa de Videoconferencias y Audiográfica, y Programa de Aprendizaje en Línea. Éstos justifican su accionar en el Plan de Trabajo 2011-2015, el cual cuenta con seis líneas de acción:

1) Creación de un Consejo Editorial de Materiales Didácticos;

2) Sistema de Gestión de Proyectos;

3) Reorganización;

4) Sobre la Relación PROMADE-Dirección Editorial;

5) Plataformas Integradas para Producir y Distribuir Contenidos en Línea, $y$

6) Radio y Televisión Educativas (Muiños Gual, 2011: 1-10).

De los cinco programas adscritos a la Dirección de Producción de Materiales Didácticos nos enfocaremos en PROMADE, instancia cuyas producciones están sujetas al sello editorial EUNED, además de las producidas por la propia editorial, así como revistas y otras publicaciones acerca de las cuales no nos ocuparemos en este trabajo.

El Programa de Producción de Material Didáctico Escrito cuenta con un coordinadoryveintiúnrecursoshumanos denominadoseditoresacadémicos.Es- 
tos profesionales cubren la oferta académica de las cuatro escuelas de la UNED y la del Colegio Nacional de Educación a Distancia (CONED) que imparte estudios de bachillerato. Los editores académicos ${ }^{2}$ vigilan la calidad académica de los materiales a través de una mediación didáctica apropiada que procureel aprendizaje autónomo, autorreguladoy autodirigido.Desdela fundación de la UNED, PROMADE ha sido el centro y eje de la producción de aquellos textos que son el principal objeto de consumo didáctico de los estudiantes de la universidad. Por su calidad, pertinencia y actualidad, los contenidos didácticos elaborados en PROMADE son ampliamente requeridos en instituciones educativas públicas y privadas de nivel medio y superior del país.

La labor de PROMADE parte de que

la edición académica cumple, más que ninguna otra, la función de incubadora y creadora de la obra que finalmente llegará a manos de los estudiantes. La semilla de toda unidad didáctica se encuentra en el diseño curricular, el plan primigenio que debe guiar la realización del material (ya se trate de un libro, un manual, una antología o una guía de estudio) y el cual debe ser cumplido en todos sus aspectos de contenidos, metodología, calidad y profundidad. Los editores académicos toman ese plan de trabajo como punto de partida y acompañan al autor durante el proceso de transformar el diseño en una obra didáctica. (“Presentación del Programa...", 2012: s. pág.)

El proceso de producción de una Unidad Didáctica Modular, a diferencia de otros materiales con fines didácticos publicados por editoriales universitarias en América Latina y el resto del mundo, evidencia un conjunto de implicaciones complejas entre academia, instancias de evaluación y tareas editoriales. El proceso inicia con la creación de la carrera y asignatura por parte de la Vicerrectoría Académica, después se hace el diseño curricular en el Programa de Apoyo Curricular y Evaluación de los Aprendizajes (PACE) ${ }^{3}$

2 Los editores académicos son coordinadores del equipo de producción encargados de garantizar el cumplimiento de la mediación didáctica en todas las dimensiones del proceso editorial. Los editores académicos son los primeros lectores y críticos de la obra: proponen cambios de estructura; ampliación y recortes de temas; aumento o disminución de contenidos; actividades complementarias y de autoevaluación; recursos para llamar la atención del estudiante; descansos en la lectura y metáforas e iconografías visuales. Su objetivo final es la creación de un entorno de lectura didáctico con herramientas facilitadoras del aprendizaje autorregulado de los estudiantes a distancia.

Una vez finalizada la versión preliminar del material escrito, el editor debe acompañar el proceso de evaluación y validación; las posibles correcciones derivadas de éste y la conclusión del material para su primera edición definitiva. Sólo entonces, cuando la obra está lista para colocarse en las librerías y/o distribuirse por la oficina institucional respectiva, se libera de la responsabilidad editorial, con lo que se finiquita su labor.

3 EI PACE es la dependencia que orienta y acompaña sistemáticamente, en materia curricular y evaluación de los aprendizajes, a los programas de extensión, pregrado, grado y posgrado de la UNED. Entre sus funciones destaca "evaluar la utilización, administración y pertinencia de los medios didácticos aplicados en los procesos de enseñanza y aprendizaje en la universidad" (Programa de Apoyo..., 2012: s. pág.). 
que lo trabaja con un especialista y con la cátedra. Cuando es aprobado y entregado al encargado o encargada de la cátedra, éste acude a PROMADE con una Solicitud de Producción. ${ }^{4}$

En PROMADE la Coordinación selecciona a un editor académico, quien convoca al equipo de trabajo a una reunión de inducción al proceso y solicita a los autores la elaboración del Plan Global (propuesta de contenidos que hace el autor sobre el diseño curricular de un curso; incluye un programa de cumplimiento). A partir de la revisión y aprobación del Plan Global, los autores inician la redacción del texto. Luego de que éstos entregan cada capítulo, el editor académico solicita al resto del equipo realizar observaciones, según su función, al trabajo elaborado. La retroalimentación es el sustento del proceso ya que, por cada capítulo, el especialista, los encargados de cátedra, los encargados de programa y el editor académico entregan observaciones que los autores deben contemplar y/o incorporar.

Posterior a la revisión de todo el equipo, autores y editor académico continúan perfeccionando cada capítulo, reenviándose versiones mejoradas hasta cumplir los objetivos trazados en el Plan Global. Después de esto, se lleva a cabo la corrección de estilo a cargo de algún miembro del equipo de filología del PROMADE. La Coordinación da el visto bueno a la obra, que se imprime en versión preliminar ${ }^{5}$ y se distribuye para su validación por la comunidad académica y estudiantil. Una vez validada esta primera versión, se solicita a la Editorial el diseño y diagramación de la UDM. El proceso concluye con la entrega oficial de la nueva unidad didáctica a la comunidad universitaria.

El Proceso de Producción Académica ejecutado en PROMADE se observa en el siguiente flujograma desprendido de la aplicación, revisión y ajustes hechos al documento "Lineamientos para la Producción de Materiales Didácti-

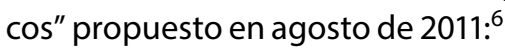

4 La Solicitud de Producción que se entrega a PROMADE es un formato implementado hace 35 años y que con el tiempo se ha ido modificando hasta tener uno más pulido. El formato contiene ocho partes: datos del material solicitado; datos de los autores; datos del especialista (sólo para Unidades Didácticas); datos de la cátedra; información de los programas que utilizan el material; datos de la autorización para la solicitud del material; información general y datos para uso interno de PROMADE. La utilidad de la Solicitud de Producción, a decir del Coordinador del Programa, "es para una mejor organización del trabajo y para ver cómo se comporta en general nuestra producción". (Gustavo Hernández Castro, entrevista realizada por la autora, julio de 2012, San José de Costa Rica)

5 Una versión preliminar de una Unidad Didáctica Modular es una edición acabada en Word que será sometida a un proceso de validación, por lo que se imprimirán únicamente la cantidad de ejemplares a utilizarse en un cuatrimestre. Es enviada a la Oficina de Distribución y Ventas de la Universidad (OFIDIVE) en formato PDF para ser reproducida. No se comercializa.

6 En 2011 la entonces Coordinadora del Programa de Material Didáctico Escrito generó el documento "Lineamientos para la Producción de Materiales Didácticos", el cual reúne todos los lineamientos acordados o establecidos concernientes a la producción de materiales didácticos escritos identificados durante su gestión. Para facilitar la identificación de su área de aplicación se organizaron según el tipo de material didáctico al que se refieren. 


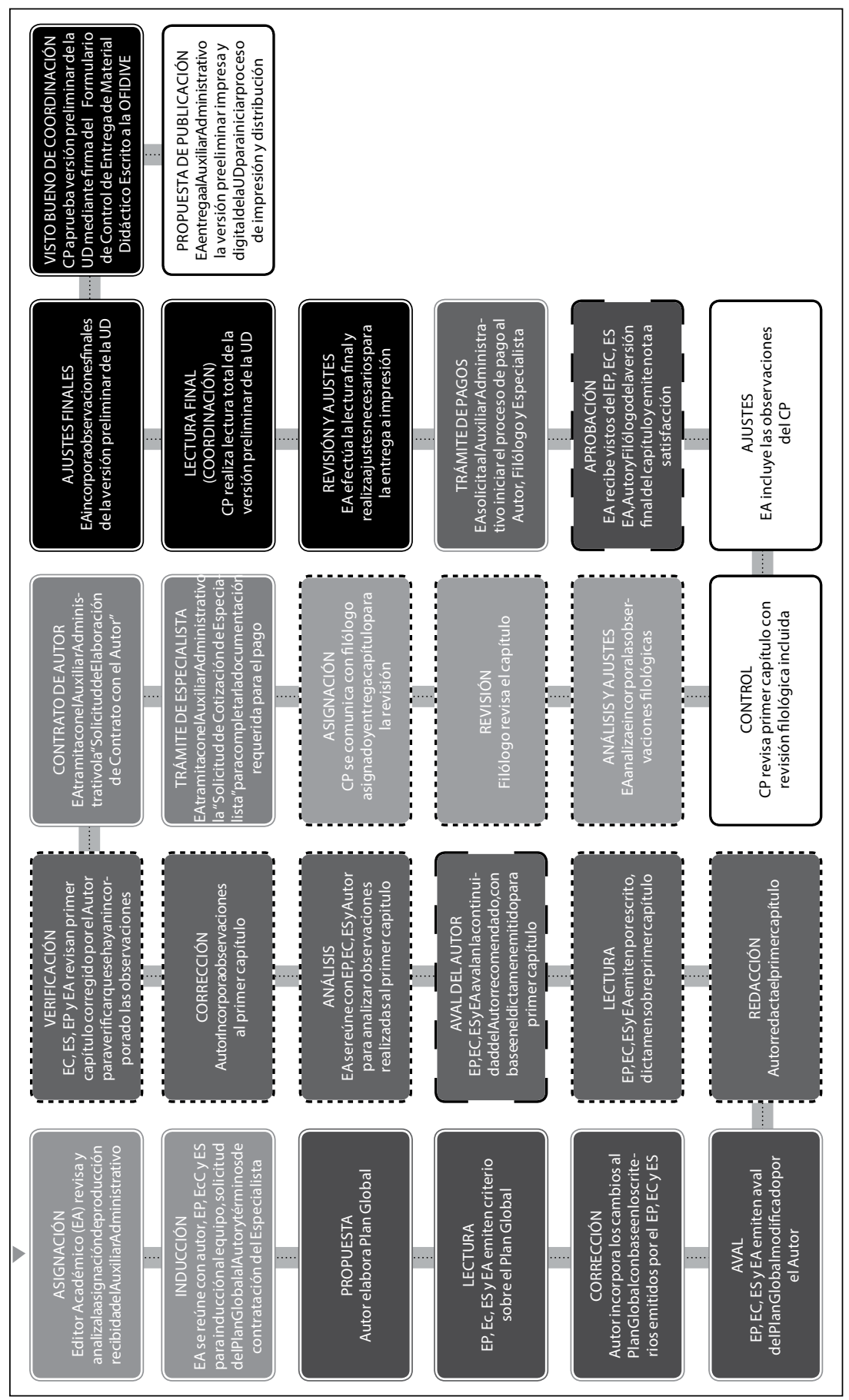




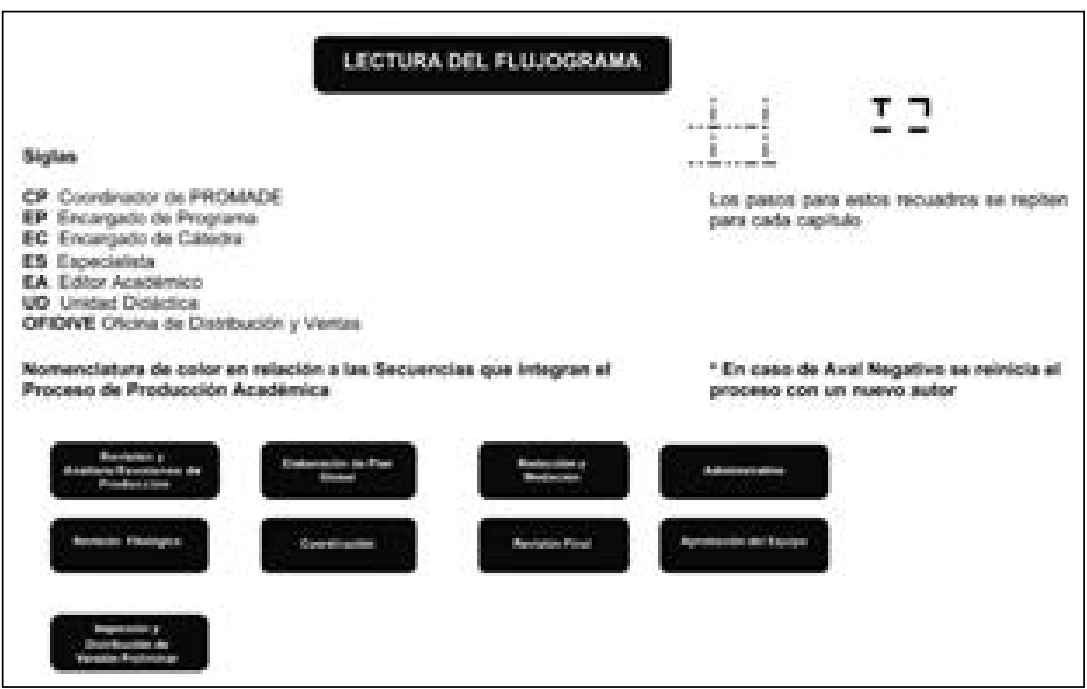

Complementa al anterior instrumento la "Descripción del Proceso de Producción Académica de PROMADE", documento que sintetiza número y nombre de secuencias, actividades, pasos, responsables, días hábiles, observaciones y documentación. Al final, se subraya que 96 es el promedio de días hábiles necesarios para la edición del primer capítulo de una Unidad Didáctica Modular; 55 los días hábiles de producción por capítulo a partir del segundo capítulo; 24 el número de pasos que demandan tiempo en días hábiles y 17 meses o 371 días hábiles el tiempo estimado de producción para una Unidad Didáctica Modular de seis capítulos y 360 páginas.

En América Latina son pocas las editoriales universitarias o programas de publicaciones que han estandarizado sus procesos editoriales más allá del simple establecimiento de una política editorial, PROMADE forma parte de estas excepciones. Durante la última etapa de gestión de la anterior coordinadora del Programa fue conformada la Comisión de Sistematización y Estandarización de la Producción de Unidades Didácticas. Esta Comisión produjo entre la segunda mitad de 2011 y abril de 2012 los siguientes instrumentos referentes a la recepción de documentos, contratación de autores y especialistas así como trámites de pagos, entre otros: ${ }^{7}$ 
- Trámite de contrato y recepción de documentos para autores.

- Procedimiento de recepción de documentos y trámite de pago de especialistas.

- Ingreso de Datos al Programa de Registro de Producciones Activas (RPA).

- Entrega de Materiales Didácticos a la Oficina de Distribución y Ven$\operatorname{tas}^{8}$ (OFIDIVE).

- Elaboración y actualización del perfil de puestos para PROMADE. Dos son los formularios que sustentan esta práctica: el Cuestionario sobre las características funcionales de los puestos de trabajo en PROMADE y la Hoja de sistematización descriptiva de los puestos de PROMADE.

- Manejo de Información Impresa Clasificada. Para la preparación de este material se tomaron como fundamentos la Ley general de control interno (Ley No 8292); el Manual de normas generales de auditoría para el sector público (M-2-2006-CO-DFOE); el Manual para el ejercicio de la auditoríainterna en las entidadesyórganossujetosalafiscalización dela Contraloría General de la República (aprobado por el Consejo Universitario de la UNED el 16 de mayo de 1986) y el Reglamento de auditoría interna (aprobado por el Consejo Universitario de la UNED el 30 de octubre de 1986).

Al ser PROMADE una oficina de la Dirección de Producción de Materiales Didácticos (DPMD) también es objeto de las disposiciones generales de esta dependencia, como la incorporación de su coordinador en la Comisión de Investigación de la Dirección de Producción de Materiales Didácticos (COMI-DPMD). Esta Comisión, creada en junio de 2011 y activa hasta la fecha (2014), tiene como atribución generar propuestas de investigación relacionadas con sus funciones como Dirección y vinculadas al quehacer de sus cinco programas. Las propuestas deben situarse en algunas de las líneas de investigación definidas por la Vicerrectoría de Investigación de la Universidad.

El peso e importancia de la producción de Unidades Didácticas Modulares (UDM) como instrumentos de difusión de conocimiento con sello editorial EUNED se visibiliza en el Informe Final de Gestión 2005-2011 de la

8 La UNED creó en agosto de 1977 la Dirección de Operaciones, primer antecedente de la hoy Oficina de Distribución y Ventas (OFIDIVE) de la Universidad. Esta oficina tiene dos objetivos generales: 1) cumplir eficientemente con la distribución de libros y cronogramas a los centros universitarios durante todo el año y 2) generar ingresos propios por medio de la venta de libros y audiovisuales. La oficina efectúa la distribución contra matrícula a los estudiantes de la universidad. Una vez que éstos cubren el costo de inscripción o reinscripción en la Sede Central o en el centro universitario más cercano a su domicilio o trabajo, reciben sus materiales del cuatrimestre (unidades didácticas, multimedios, audiovisuales, etcétera). 
Dirección Editorial. En este documento el director ejecutivo reporta que de 2005 a 2010 se produjeron 2534 Unidades Didácticas Modulares (títulos), equivalentes a 1166431 ejemplares o 592790717 de páginas. Su impresión tuvo un costo de 2102742028.18 colones, lo que constituye el $69.35 \%$ de los egresos totales de la EUNED. Las UDM representaron el $83.14 \%$ del total de la producción editorial catalogada como "libros" durante este periodo. ${ }^{9}$ El citado informe también comunica que las producciones más costosas por página son los libros de línea editorial (9.66 colones) y las revistas (8.03 colones) en contraste con las Unidades Didácticas Modulares (3.55 colones) (Muiños Gual, 2011: 4, 14).

Por otra parte, el Informe de Labores de la Dirección de Producción de Materiales Didácticos: Julio 2011 a Julio 2012 y Propuestas de Acciones hasta el 2015 (Román González, 2012: 6) destaca el hecho de que PROMADE continúa siendo el centro de atención de la producción didáctica en la UNED pues, al 30 de junio de 2013, la cantidad de producciones solicitadas a este programa por escuela en contraposición a los restantes cuatro era el que se muestra en la Tabla 1.

Tabla 1. Cantidad de producciones solicitadas por escuela a cada uno de los cinco programas de producción de materiales didácticos

\begin{tabular}{|c|c|c|c|c|c|c|}
\hline Escuela & PROMADE & $\begin{array}{c}\text { PAL } \\
\text { (Programa de } \\
\text { Aprendizajeen } \\
\text { Línea) }\end{array}$ & $\begin{array}{c}\text { PPMA } \\
\text { (Programa de } \\
\text { Producción } \\
\text { de Material } \\
\text { Audiovisual) } \\
\text { (Horas } \\
\text { Producción) }\end{array}$ & \begin{tabular}{|c} 
PEM \\
(Programa de \\
Producción \\
Electrónica \\
Multimedial)
\end{tabular} & $\begin{array}{c}\text { VAU } \\
\text { (Programa } \\
\text { de Videocon- } \\
\text { ferencias y } \\
\text { Videográfica) }\end{array}$ & $\begin{array}{c}\text { Total por } \\
\text { Escuela }\end{array}$ \\
\hline $\begin{array}{l}\text { Escuela de } \\
\text { Administración } \\
\text { (ECA) }\end{array}$ & 82 & 49 & 0.0 & 0 & 21 & 152.0 \\
\hline $\begin{array}{l}\text { Escuela de } \\
\text { Ciencias de } \\
\text { la Educación } \\
\text { (ECE) }\end{array}$ & 24 & 258 & 9.5 & 8 & 17 & 316.5 \\
\hline $\begin{array}{l}\text { Escuela de } \\
\text { Ciencias } \\
\text { Exactasy } \\
\text { Naturales } \\
\text { (ECEN) }\end{array}$ & 130 & 439 & 124 & 14 & 27 & 734 \\
\hline
\end{tabular}

9 La Producción Editorial en la EUNED trabaja dos rubros: libros (Unidades Didácticas Modulares, línea editorial, otros libros) y otros materiales (materiales para matrícula, revistas y otros trabajos de impresión). 


\begin{tabular}{|l|c|c|c|c|c|c|}
\hline $\begin{array}{l}\text { Escuela de } \\
\text { Ciencias } \\
\text { Sociales } \\
\text { Humanidades } \\
\text { (ECSH) }\end{array}$ & 79 & 354 & 14.8 & 11 & 42 & 500.8 \\
\hline $\begin{array}{l}\text { Total por } \\
\text { programa }\end{array}$ & $\begin{array}{l}365 \\
{[\mathrm{sic}]}\end{array}$ & 1100 & 148 & 33 & 107 & 1753 \\
\hline
\end{tabular}

La DPMD produce desde 2009 el Análisis estadístico de materiales didácticos para la oferta académica del Programa Académico (PAC), que permite conocer anticipadamente el comportamiento de la demanda de materiales didácticos para un determinado ciclo lectivo o escolar. Los aspectos considerados en este análisis son: 1) Oferta académica del PAC en curso; 2) Registro de Producciones en el Programa de Registro de Producciones Activas (RPA) para el Colegio Nacional de Educación a Distancia (CONED), y 3) Registro de producciones en el RPA para el Sistema de Estudios de Posgrado (SEP).

A partir de enero de 2012 se sumó una nueva herramienta que ayuda a controlar con mayor eficiencia los materiales didácticos solicitados por Escuela y Cátedra en la UNED, ésta es la Sábana de Producción de Material Didáctico. La Sábana analiza los pedidos de más de 90 jefes de Cátedra y oficinas de la institución mediante tablas de Excel. El programa consta de dos partes: la primera presenta gráficas de cada programa de la DPMD y la segunda muestra una línea que representa todos los servicios demandados por un curso en concreto. A esta herramienta tienen acceso los Directores de Escuela, el Director de la DPMD, los jefes de los cinco programas de la Dirección y la Vicerrectoría Académica. Sin embargo, la Sábana no concentra todo lo que hace cada programa porque no están integrados el SEP, el CONED, la Dirección de Extensión, Centro de Idiomas, Agenda Universitaria y Agenda Joven, instancias que también hacen solicitudes a la Dirección.

En el apartado de PROMADE del Informe de Labores de la Dirección de Producción de Materiales Didácticos: Julio 2011 a Julio 2012 y Propuestas de Acciones hasta el 2015 se menciona que al momento de la redacción del mismo (junio de 2012) el Programa trabajaba para concluir:

- Manual de Procedimientos Administrativos en concordancia con los requerimientos de Control Interno y de la Normativa Internacional de Calidad (ISO).

- Módulo del Manual de Estándares de Publicación (Manual de Estilo) y Módulo de Tipografía y Ortotipografía, este último en proceso de validación y consulta con la comunidad universitaria (Román, 2012: 6). 


\section{Metodología}

La edición universitaria es concebida, por sus características, como industria cultural, campo cultural, industria creativa e industria de contenidos. En el presente texto se aborda desde la categoría de industrias de contenidos. Las industrias de contenidos comprenden la producción pensada para las nuevas tecnologías de la información y comunicación, la convergencia digital y plantean la inclusión social. Es decir, abarcan nuevos modelos de negocio, como losconglomeradosmultimedia, ${ }_{10}^{10}$ nuevasestructurastecnológicas,nuevoslenguajes para esos medios digitales, nuevas maneras de relacionarse con los diferentes públicos y nuevos profesionales capacitados para nuevas demandas, los denominados nuevos intermediarios culturales. ${ }^{11}$

Las industrias de contenidos son planeadas y desarrolladas independientemente de tiempo, espacio o localización geográfica; incluyen la interactividad y movilidad posibilitadas por telefonía móvil, computadoras portátiles, tablets, etc. Dicho conjunto de acciones ocurre en el ámbito editorial con la publicación de textos en sitios web especializados, la concertación de ventas de derechos de autor vía correo electrónico, la diversificación de formatos como el ebook, ePub, la impresión bajo demanda (IBD) y el marketing en redes sociales. Sostiene la investigadora en comunicación y periodismo Cosette

10 Los conglomerados multimedia son producto de las fusiones y alianzas estratégicas entre empresas dedicadas a la cultura, la información y el entrenamiento localizadas en diferentes países. Una característica fundamental de estos grupos de medios es que pasaron de ser instrumentos de difusión del Estado y de grupos capitalistas a imponer su agenda política y económica. El lector interesado en abundar sobre el tema puede consultar a Guerra González (2009).

11 Las nuevas condiciones socioeconómicas de la última década del siglo XX obligaron a concebir procesos culturales como el de la edición en un contexto de mercado, requiriendo para su análisis de nuevos conceptos y marcos de explicación. El norteamericano Mike Featherstone retoma afinales delosnoventala categoríabourdiana deintermediariosculturales anteponiéndole el adjetivo de nuevos, con lo cual estaría describiendo a un conjunto de nuevas profesiones vinculadas con la producción simbólica del capitalismo tardío en el marco de las transformaciones sociales oriundas de la crisis del Estado de Bienestar, los nuevos movimientos políticos y, obviamente, el posicionamiento del mercado como el regulador único del horizonte de la vida cotidiana y del surgimiento de lo que autores como el chileno Oscar Mac-Clure y el español Ludolfo Paramio dan en llamar las nuevas clases medias.

Los nuevos intermediarios culturales son agentes que hacen circular rápidamente información entre áreas de la cultura antes cerradas (los campos culturales). En una sociedad que se orienta hacia el consumo de mercancías simbólicas y en donde cobran importancia el gusto y el juicio discriminatorios, el capital de conocimientos o de cultura habilita a grupos o a categorías particulares de personas a comprender y clasificar los nuevos bienes de manera apropiada y mostrar cómo usarlos a una amplia gama de audiencias y consumidores (Featherstone, 2002: 55-56). Los nuevos intermediarios culturales son, en el espectro de la industria editorial comercial, los individuos e instituciones que condicionan y direccionan la oferta de publicaciones que han de ser objeto de consumo por parte de los lectores, entendiéndose por éstos a los recientes actores involucrados en la cadena del libro (agentes literarios, traductores, empresas digitalizadoras de contenidos, bibliotecas digitales, artistas gráficos, compradores institucionales, etcétera). 
Castro que "lo que marca posición en defensa de esta categoría es la posibilidad de participación ciudadana, así como la inmensa carga de informaciones que los habitantes del orbe podrán recibir diariamente (aparte de las que reciben por medio de impresos y audiovisuales)" (Castro, 2008: 15-18).

La categoría de industrias de contenidos es trabajada a detalle por los especialistas de la Economía de la Cultura, sector de la economía dedicado a definir políticas y estrategias para productos que tienen, al mismo tiempo, potencial económico y valor simbólico (mensajes, identidad, valores). La Economía de la Cultura estudia la generación de propiedad intelectual y derechos de autor, elemento fundamental del negocio del libro, y un derivado de éstos, los derechos subsidiarios. ${ }^{12}$ Es ampliamente utilizada como disciplina medular en la Gestión y el Desarrollo Cultural a nivel global; ha sido estudiada y aplicada en Latinoamérica por Xavier Huamayave, Ernesto Piedras Feria, Néstor García Canclini y Ana Carla Fonseca.

En el análisis de la dimensión económica de la cultura residen dos puntos de vista contrapuestos. El primero de ellos, vinculado al libre mercado, considera que aunque las industrias culturales cuentan con personalidad propia, comparten características con otros sectores económicos y como tal tienen que ser tratadas. La segunda perspectiva, alineada con la defensa de la gestión pública del arte y la cultura, supone que este sector, aunque de gran importancia económica, no puede ser tratado comolos demásya que entraña repercusiones de índole social y humano que han de primar sobre los aspectos pecuniarios. La consideración de la cultura como patrimonio de la humanidad mora en esta vertiente y es afín a las tareas de la universidad pública. ${ }^{13}$

En el caso de las industrias de contenidos insertas en la convergencia digital, los economistas de la cultura consideran los indicadores siguientes: 1. Conocimiento; 2. Inmediatez; 3. Digitalización; 4. Virtualización; 5. Desintermediación; 6. Interconexión en red; 7. Innovación y 8 . Nuevo rol de los actores.

Al emplear esta metodología se responde a preguntas como:

a) ¿Cuál es el impacto del conocimiento producido y/o difundido en la UNED al considerar su originalidad, destinatario, tipo (académico o científico), carácter (público o privado), etcétera?

b) ¿En cuáles procesos editoriales de la UNED se presenta el valor inmediatez?

12 Los derechos subsidiarios consisten en la venta de un producto cultural, su contenido e incluso sus personajes a cineastas, empresas de ediciones populares, clubes de libros, ediciones extranjeras y fabricantes de, por ejemplo, camisetas, carteles, tazas y tarjetas de felicitación (Cole, 2003: 113).

13 Base de datos de la Asociación de Gestores y Técnicos Culturales de la Comunidad de Madrid, http://www.agetec.org/ageteca/economia.htm 
c) ¿Cómo es asumida la digitalización en el caso de estudio seleccionado en cuanto a hardware, software, recursos humanos especializados, tipos de publicaciones digitales, presencia de retrodigitalización, diversificación de contenidos y derechos de explotación para diferentes medios?

La metodología empleada es de carácter cualitativo y da cuenta de las dinámicas institucionales de un programa editorial que no por ser de carácter educativo deja de estar influido por lo que acontece en el sistema editorial global. De esta manera se describe brevemente el valor a cualificar y a continuación se detalla cómo es asumido en PROMADE.

\section{Indicador Conocimiento}

Según María Alejandra Tejeda Gómez, algunos de los criterios que se emplean para cuantificar el impacto del conocimiento son:

- ¿Este conocimiento se produce o se reproduce?

- ¿Para quién se produce este conocimiento?

- ¿El conocimiento es académico o científico?

- ¿Público o privado?

- ¿Local o nacional?

- ¿Regional o mundial?

- ¿Se democratiza este conocimiento (Open access) o se restringe (copyright)? (Tejeda Gómez, 2009: 305)

Según estos criterios puede decirse que el conocimiento generado en PROMADE con sello EUNED es "original", al producirse por encargo de una instancia académica acorde a las necesidades de un desarrollo curricular y cuya validez y pertinencia para los estudiantes inscritos en la Universidad Estatal a Distancia se corrobora institucionalmente con la aplicación de dos formularios de evaluación. Con el primero de ellos, PROMADE evalúa el uso que hacen los estudiantes del material didáctico impreso, con el fin de mejorar las propuestas didácticas acordes con el modelo pedagógico de la UNED. El segundo formulario se aplica para que los tutores evalúen los materiales didácticos escritos. La información obtenida es confidencial y para uso exclusivo de la institución.

Por restricciones de la Ley de Derechos de Autor, menos del $5 \%$ del material que produce PROMADE se encuentra en acceso gratuito a través de la Plataforma en Internet de Recursos Didácticos (http://recdidacticos.uned. 
ac.cr/). Sin embargo, el precio de venta al público de las Unidades Didácticas Modulares no sobrepasan los 10000 colones $^{14}$ (20 USD aproximadamente), y contribuye a que sean adquiridas por un mayor número de lectores, lo que impulsa la democratización del conocimiento.

El precio de venta de las Unidades Didácticas Modulares se fija a partir de los costos de edición, impresión, distribución y derechos de autor. Con base en estos factores se establece un factor de multiplicación que deja un margen de ganancia de $1.75 \%$, que es casi imponderable. El gasto previo es un gasto que la UNED considera como una subvención estatal.

\section{Indicador Inmediatez}

En la economía tradicional un nuevo invento aseguraba una corriente de ingresos durante décadas. En la nueva economía, la inmediatez es una propiedad fundamental, generadora de competencia y oferta de nuevos servicios en todos los sectores, sea en el campo de la comunicación o en la medicina. Al considerar que Internet ha ido modificando las relaciones entre las personas y empresas, porque facilita el intercambio inmediato de conocimiento y servicios, la obsolescencia es la variable a evitar. En el caso de la edición de libros destinados a los procesos de enseñanza-aprendizaje, como es el de las Unidades Didácticas Modulares de PROMADE, su elaboración y actualización constante se han estandarizado en un esfuerzo de planificación continuo para evitar que lleguen al estudiante cuando ya son obsoletos. Para ello se han diseñado herramientas de control de la producción como la Sábana de Producción de Material Didáctico, de la que se trató con anterioridad y que transita para trocarse en un software analítico y estadístico de la producción y gestión editorial académica.

De acuerdo con esa línea, es crucial fortalecer el Portal de Recursos Didácticos para potenciar la inmediatez de los contenidos académicos del Programa. Se torna indispensable mantener actualizados los contenidos de dicha plataforma en línea para que puedan ser consultados fácilmente tanto por estudiantes de la UNED como por personas externas a la universidad. La iniciativa no ha terminado de concretarse porque el contrato de edición del Programa todavía no establece la divulgación de las obras en variedad de soportes, total o parcialmente. Incluso en el Informe de Labores de la Dirección de Producción de Materiales Didácticos: Julio 2011 a Julio 2012 y Propuestas de Acciones hasta el 2015 se menciona como una de las metas para dicho periodo el que:

14 Un texto didáctico para educación superior de una editorial comercial se encuentra en el mercado nacional en 17000 colones (34 USD aproximadamente) en precio de descuento. 
Se requiere cambiar el paradigma existenteen la relación contractual delos autores con la UNED. Actualmente funciona un único formato de contrato (por encargo de obra), que limita las posibilidades a PROMADE y a la Academia de contar con contenidos entregados según cronogramas de trabajo, o bien de utilizar los contenidos redactados por autores, por ejemplo, un autor que escribe cuatro capítulos de un total de seis y después comunica que no continuará con la producción, deja a PROMADE y a la Academia sin posibilidad de emplear los contenidos ya escritos y pagados. Para solventar lo anterior, se considera contar con estas opciones contractuales: a) Contratos por encargo de obra (ya existente); b) Contratos por compra de contenido; c) Contratos por obra colaborativa, entre otros. (Román González, 2012: 6. Cursivas desde el original.)

\section{Indicador Digitalización}

Anteriormente la información era analógica y física, por fuerza necesitaba del desplazamiento de las personas a salas de reuniones, requería el uso de efectivo o cheques, presentaba señales de televisión y radio analógica, línea telefónica analógica, precisaba del envío de cartas, archivos impresos, etc. En la nueva economía, los archivos de imágenes, texto, sonido y datos son digitales, las reuniones se efectúan de forma virtual, los cheques y el dinero en efectivo son sustituidos por tarjetas de crédito y métodos para hacer y recibir pagos como PayPal.

En el ámbito de la edición existen dos tipos de publicaciones digitales: un primer grupo corresponde a las reproducciones de los impresos tratados como imágenes gracias a programas informáticos como Adobe Acrobat System (Ios archivos PDF). Éstos permiten a editores y libreros ofrecer al público el mismo contenido, con idénticas características de forma que el libro o la revista en papel; sólo se altera el soporte de lectura (computadora, tablet, etc.). El sistema tiene entre sus ventajas la retrodigitalización, proceso que admite digitalizar, mediante un escáner óptico, el impreso original y conservar este archivo informático, completo o por partes, para su resguardo permanente y su comercialización o difusión por diferentes canales digitales.

El segundo grupo o tipo de publicaciones digitales guarda relación con las nuevas posibilidades y desafíos que devienen de las innovaciones en software y en soportes de lectura; incluye aquéllas que pueden consultarse en la web o en dispositivos móviles, como el libro electrónico.

Ejemplo de los esfuerzos encauzados a la digitalización de la producción de conocimiento con sello EUNED es el Portal de Recursos Didácticos de la Dirección de Producción de Materiales Didácticos, que permite la publicación de textos en formatos digitales como PDF, SWF y ePub. A marzo de 2014 se localizaban en este portal 129 documentos sobre temáticas de 13 discipli- 
nas; una versión preliminar de una Unidad Didáctica Modular y 9 materiales de consulta, además de producciones de los restantes cuatro programas de la DPMD.

A su vez, la digitalización permite la impresión bajo demanda, que ahorra costos debido a que pueden imprimirse tirajes cortos de revistas, libros y papelería de corte institucional (folletos, carteles, etc.). En el Informe Final de Gestión 2005-2011 de la Dirección Editorial (Muiños Gual, 2011: 27) se destacan como ventajas comparativas de la impresión digital bajo demanda versus el offset las que se mencionan a continuación:

a) Permite realizar cambios en el contenido de un tiraje a otro sin costos de materiales, lo cual es determinante para la actualización o puesta a prueba de los materiales didácticos.

b) Evita la acumulación de inventarios y el riesgo de pérdidas por obsolescencia de los textos.

c) Las nuevas tecnologías de impresión digital en línea permiten realizar con una sola operación desde la computadora las operaciones de impresión, compaginado, doblado y encuadernado de folletos y libros.

d) Es una tecnología limpia y amigable con el ambiente.

Durante el periodo 2005-2010 la UNED hizo una inversión de 371133948 colones (743 000 USD aproximadamente) para la adquisición de tecnologías de impresión digital. Una vez que se analizó la eficiencia de estos nuevos equipos por dos años pudo establecerse 1) una estrategia de producción sustentable de los materiales didácticos que considera cinco variables (tiraje, uso del color, capacidad de producción, cantidad de páginas y vida útil del material) y 2) políticas de producción a nivel institucional (Muiños Gual, 2011: 30-31). Esta inversión también permite a la EUNED comercializar servicios de su Taller de Impresión de manera externa.

Para el periodo 2011-2014 la EUNED proyectó un plan de inversiones en este rubro en el marco del préstamo ${ }^{15}$ del Banco Interamericano de Desarro-

15 En 2009 CONARE presentó a los ministros representantes del Poder Ejecutivo del Gobierno de Costa Rica el Proyecto de Mejoramiento de la Educación Superior para un convenio de financiamiento de las universidades públicas que integran este Consejo con el Banco Mundial. El convenio es una operación de crédito por 200 millones de dólares que se distribuirían en iguales proporciones entre las cuatro universidades con la intención de concretar estas metas: 1) elevar su admisión de estudiantes y ampliar los cupos en las carreras que lo requieran; 2) reforzar su capacidad científico-tecnológica expandiendo la infraestructura física, el equipamiento, las becas a profesores y la ampliación de los servicios estudiantiles incluyendo residencias. Tales metas se relacionan directamente con la orientación del Plan Nacional de Educación Superior Universitaria Estatal (PLANES), previsto para el periodo 2011-2015. 
Ilo (BID) al Consejo Nacional de Rectores (CONARE) y las universidades que lo integran. Según proyecciones de su Director Ejecutivo, la inyección económica en equipo digital a blanco y negro, escáneres para edición y preprensa, software de administración de la producción y cámara fotográfica pondrá a la EUNED y a su Taller de Impresión a la cabeza de la edición en Costa Rica (Muiños Gual, 2011: 61).

Por su parte la DPMD consignó en su Informe de Labores de la Dirección deProducción de Materiales Didácticos: Julio 2011 a Julio 2012 y Propuestas de Acciones hasta el 2015 como asunto prioritario la renovación del equipo tecnológico (computadoras):

En los últimos cinco años a PROMADE se le ha asignado equipo de segunda mano que han desocupado el PEM y el PAL. La solicitud de cambio se realizó el 16 de febrero de 2012 a las Vicerrectorías Académica y Ejecutiva. Con este cambio la academia se fortalecería en: 1) interacción en línea con programas de edición que permitan comunicarse en tiempo real con los autores de contenido y 2) actualización de los materiales didácticos en línea sin tener que realizar gastos excesivos en impresión de papel.

La renovación del equipo, además de lo señalado, permitirá a PROMADE, la DPMD y a la institución contar con una publicación (revista digital) especializada, que sea punto de encuentro y sistematización de más de 30 años de experiencia de producción de materiales didácticos. Hoy las universidades del mundo están fortaleciendo la edición de textos académicos con estos espacios para la reflexión. (Román González, 2012:6)

La producción del ebook está pendiente porque la Universidad no ha resuelto lagunas legales en relación a los derechos de autor de los contenidos. Sin embargo, como el proyecto fue aprobado por el Consejo Universitario en 2011, en agosto de 2012 iniciaron la impartición de capacitaciones sobre el tema dirigidas a personal de EUNED, PROMADE y OFIDIVE a través de la contratación de cursos con empresas latinoamericanas como Versal (México).

\section{Indicador Virtualización}

La virtualización facilita contar con una extensión de la institución para difundir, promocionar y comercializar los servicios o productos a los que ésta se dedique. Este aspecto es contemplado por PROMADE y la EUNED en la figura de la librería virtual que está diseñando el área de Ventas por Internet de la OFIDIVE. Originalmente se pretendía arrancar con este proyecto en el segundo semestre de 2013 para la venta de libros físicos y ampliar el espacio para la comercialización de libros electrónicos en 2015, pero debido 
a factores legales y tecnológicos, en mayo de 2014 la librería virtual seguía sin ponerse en marcha en ninguna de las dos modalidades.

\section{Indicador Desintermediación}

En las redes digitales varias funciones de intermediación dejan de existir. La principal ruptura se produce en la distribución, tradicionalmente controlada por las grandes empresas (gatekeepers ${ }^{16}$ ) que en numerosos sectores representaban un embudo por el cual debían pasar las iniciativas ávidas de alcanzar el mercado. Con el desarrollo de Internet las nuevas empresas e instituciones de menor tamaño saltan los canales de distribución tradicionales. Lo que inicialmenteera una ventaja estratégica para las grandes empresas puede convertirse en su principal problema porque han de mantener distintos canales de distribución con redes muy costosas que, sin embargo, no les garantizan el control del mercado. En contraste, las nuevas empresas aparecidas en la era de Internet logran distribuir a costos muy reducidos y sin estructuras fijas (Casani, Rodríguez-Pomeda y Sánchez, 2012: 56).

La desintermediación de los materiales de PROMADE es limitada, continúan distribuyéndose por canales tradicionales como librerías físicas. Además de la distribución contra matrícula la OFIDIVE realiza distribución en librerías de administración propia a cargo de la Supervisión de Librerías; librerías de administración de los centros universitarios y librerías comerciales privadas (labor a cargo del Promotor de Ventas).

Las librerías de administración propia son cuatro (Mercedes de Montes de Oca, San José Centro, Heredia Centro y Cartago Centro). Existe una quinta librería en convenio con el Ministerio de Obras Públicas y Transportes (MOPT) en la que se venden el Manual del Conductor, Manual de Transporte Público, CD del Manual del Conductor y algunos títulos de línea editorial. Estos establecimientos cuentan con dos dependientes y un administrador, quien reporta directamente al Supervisor de Librerías a través de un sistema de facturación que se actualiza diariamente.

Es importante señalar que las librerías de la universidad no sólo comercializan libros y revistas de la EUNED sino también de otras universidades y editoriales. Sin embargo, debido al crecimiento exponencial de su fondo ${ }^{17}$ se ha ido reduciendo el espacio destinado a la exhibición de materiales externos al punto de que las librerías sólo aceptan en consignación aquéllos que tienen una buena rotación.

16 Un análisis sobre el papel de los editores universitarios como guardianes del conocimiento lo podemos encontrar en McGinty (1999).

17 El fondo editorial vivo o activo de la EUNED en sus librerías es de 1180 títulos. 
Del segundo tipo (librerías de administración de los centros universitarios), una se ubica en Cañas (Guanacaste) y otra en Alajuela. En éstas el centro universitario se encarga de poner al personal y un espacio físico de 20 a $25 \mathrm{~m}^{2}$ para la exhibición de los textos, además de administrar la caja y los asuntos gerenciales. Solicita a la OFIDIVE sólo libros en consignación y asesoría para la gestión de la librería por medio de la Supervisión de Librerías. Esta estrategia pretende concretar a mediano plazo el plan de regionalización del fondo editorial de la universidad.

Uno de los obstáculos con el que topó OFIDIVE en el pasado para la comercialización de sus materiales fue que el aparato legal de la UNED no permite dar crédito a los compradores interesados en éstos. Para atenuar esta traba ahora los trámites de compra y venta al extranjero de mayoreo se hacen vía la Fundación de la Universidad Estatal a Distancia para el Desarrollo y Promoción de la Educación a Distancia (FUNDEPREDI) que tiene como misión "facilitar la gestión y la búsqueda de financiamiento para promover el desarrollo de la investigación, la docencia, la extensión y la prestación de servicios vinculados con las actividades de la UNED". ${ }^{18}$ El papeleo para compras y envíos al extranjero con la intermediación de la Fundación ${ }^{19}$ tarda 15 días hábiles y se contrata para la mensajería a Correos de Costa Rica o DHL. Las compras individuales de clientes extranjeros se gestionan hasta el momento con la empresa Mall506, ${ }^{20}$ un consorcio costarricense que arrienda locales virtuales a terceros para actividades comerciales, promocionales o de cualquier otro tipo.

Para las ventas nacionales de mayoreo la OFIDIVE trabaja conjuntamente con la Unidad de Crédito y Cobro de la Oficina de Tesorería de la Universidad, que está autorizada para dar crédito y recaudar los recursos por ventas de libros. El crédito se adecua a las necesidades del cliente y éste pude ser a 8,15 y 30 días que es el mayor plazo; OFIDIVE aplica tres modalidades de pago: de contado, crédito y consignación. Pese a esta aparente flexibilidad, el Supervisor de Librerías señaló en lo concerniente a los descuentos que "el límite de descuento según el estatuto es de $30 \%$. Sí se puede hacer un descuento mayor pero ya correspondería hacer una solicitud a una instancia ma-

18 Fundación de la Universidad Estatal a Distancia para el Desarrollo y Promoción de la Educación a Distancia, http://fundepredi.org/Quienes-Somos.html

19 Además de la UNED, las otras tres universidades estatales costarricenses también venden servicios editoriales y de otra índole a través de sus respectivas fundaciones, que llevan por nombre FUNDAUNA (Fundación para el Desarrollo Académico) en el caso de la Universidad Nacional; FUNDEVI (Fundación de la Universidad de Costa Rica para la Investigación) de la UCR y la Fundación Tecnológica de Costa Rica (FUNDATEC) en el caso del Instituto Tecnológico de Costa Rica. 
yor a la Jefatura de OFIDIVE para estos tipos de descuento. Ya hay que tocar a un Vicerrector para que él lo apruebe". ${ }^{21}$

La normativa que regula la comercialización de la producción editorial interna y externa de la UNED es el Reglamento de Ventas de Materiales Producidos y Adquiridos por la UNED, el cual fue aprobado por el Consejo Universitario el 31 de mayo de 2011. Su artículo $10^{\circ}$ referente a los descuentos se modificó el 11 de julio de 2003. Este reglamento se acompaña del Procedimiento para Crédito y Cobro del Material Didáctico Producidoy Adquirido por la UNED (17 de junio del 2000) que pone en práctica la Unidad de Crédito y Cobro de la Oficina de Tesorería.

La OFIDIVE trabaja mediante un plan quinquenal dotado de un presupuesto ajustable anualmente de acuerdo a las necesidades detectadas en sus diferentes áreas. No cuenta con un departamento de marketing y una más de sus funciones es la compra de libros externos que, por razones de tiempo o especialidad de la materia, no pueden producirse en PROMADE y son solicitados por la Academia.

Indicadores del Informe Final de Gestión 2005-2011 de la Dirección Editorial (Muiños Gual, 2011: 19) revelan que la compra del libro externo se incrementó de 2007 a 2008 de 322608948.21 colones a 454953067.44 colones, mientras que en 2009 se incorporaron 40 libros externos nuevos y sólo 11 Unidades Didácticas Modulares.

OFIDIVE señala que tres cuartas partes del tiempo de la EUNED y sus funcionarios se dedica a surtir la demanda de materiales de la matrícula. El movimiento de libros de línea editorial en correlación con las Unidades Didácticas Modulares es exclusivamente de $25 \%$. Las UDM de Ciencias Exactas son las de mayor venta porque son requeridas por estudiantes de la Universidad Nacional, la Universidad de Costa Rica y las universidades privadas del país.

\section{Indicador Interconexión en Red}

No hay presencia en redes sociales de PROMADE. De ahí que la llamada interconexión de integración, definida por la conjunción de las funciones de producción y consumo en los propios participantes (Casani, Rodríguez-Pomeda y Sánchez, 2012: 60), esté aún en pañales en este programa editorial. Una causa que incide directamente en esta situación es el control institucional sobre la información de las distintas dependencias, escuelas y programas de la UNED que antes de ser difundida en la Red debe pasar por la Oficina 
Institucional de Mercadeo y Comunicación y posteriormente por la Dirección de Tecnología, Información y Comunicaciones.

\section{Indicador Innovación}

Es la palabra clave en todos los sectores de la economía para la convergencia digital. En términos de industrias de contenidos, la responsabilidad de las empresas es concebir productos innovadores para todo tipo de medios, debido a que las posibilidades creativas de esas industrias son mucho más grandes que las presentes en los medios analógicos. La creatividad entendida como habilidad y talento individual con potencial para crear riqueza y empleo a través de la generación y explotación de la propiedad intelectual (Piedras, 2008: 29) es la principal fuente de valor en la nueva economía y la materia prima de la innovación. Así, la industria editorial se ve condicionada a innovar en adquisición de derechos, soportes, formas de comercialización y distribución si quiere sobrevivir y ser competitiva. Ejemplo de esto es la "caza de autores" en redes sociales como Facebook o Twitter emprendida por editoriales como Intangible o Sinerrata o el Twitter Fiction Festival organizado por Twitter en noviembre de 2012 ("Editoriales buscan...", 2012; Wolford, 2012).

La convergencia digital plantea a instituciones y empresas la responsabilidad de concebir servicios y productos innovadores. PROMADE ha innovado en el aspecto de distribución de materiales didácticos con la creación del Portal de Recursos Didácticos en línea con los alcances y limitantes antes descritos.

\section{Indicador Nuevo Rol de los Actores}

Hay una fuerte tendencia a la individualización en esta nueva era por medio de acciones personalizadas. Simultáneamente la convergencia digital, si es pensada desde la inclusión social, viabiliza que las audiencias y los diferentes grupos sociales hagan la transición a productores de contenidos. El tránsito de la industria del libro tradicional a una de contenidos conlleva una cadena o ecosistema editorial con menor intermediación y mayor especialización de los eslabones productivos. Así, en la UNED hablamos de la inclusión de sus académicos como editores de Unidades Didácticas Modulares (PROMADE) mientras que la participación de intelectuales locales y nacionales se concentra en la autoría de obras de la línea cultural. Es notoria la disminución en la participación de creadores independientes y del público en general en funciones relacionadas con la generación y divulgación de contenidos. 
Entre las metas para el periodo 2013-2015, PROMADE pretende:

... Formalizar, através dela oficina de recursoshumanos, elárea decorrección de estilo de PROMADE. El oficio de corrector de estilo es una especialización que es menester reconocer en la institución. En este momento las funciones de corrección se equiparan con el de editor académico, sin tener un perfil y funciones para lo que en la práctica se ejecuta en PROMADE. El equipo de filología de PROMADE, además de revisar la totalidad de los materiales didácticos, da soporte de corrección de estilo al resto de programas de la Dirección, al Sistema de Estudios de Postgrado, al Consejo Universitario, a la Oficina de Comunicación y Mercadeo y a otras áreas de la Universidad.

Por lo anterior se solicitará a la Oficina de Recursos Humanos un estudio para que oriente el nuevo perfil, competencias y funciones para la creación del puesto de corrector de estilo en PROMADE y en la Dirección. Un proceso similar se llevó en la Editorial para crear el puesto de Editor Filológico para las obras de línea editorial. [...]

... Serequierefortalecerelequipodeediciónexternaparaincrementarlaproducción de materiales didácticos (guías de estudio, manuales de laboratorio, materiales complementarios, otros) con el objetivo de que los editores académicos de planta puedan enfocarse exclusivamente en las unidades didácticas. (Román González, 2012: 11-12. Las cursivas son nuestras.)

La EUNED, como todas las dependencias y programas de la UNED y de la administración pública costarricense, efectúa su planificación anual por medio del Plan Operativo Anual (POA). ${ }^{22}$ El POA de la EUNED del periodo 2005-2011 tuvo como objetivo general "elaborar, producir y distribuir materiales didácticos impresos, audiovisuales y asistidos por computadora que requieren los programas docentes, de extensión y posgrado de la Universidad" (POA, 2011: 6). Asimismo, dio seguimiento a 10 objetivos específicos, que a su vez contenían Metas programadas, Unidad de medida, Avance del cumplimiento y Observaciones relevantes con relación al cumplimiento de las metas semestrales. De los 10 objetivos, 5 alcanzaron un cumplimiento de $100 \%$ o más:

1. Producir y reproducir los materiales educativos impresos para los estudiantes de la UNED (184\%).

22 El POA es la guía fundamental de gestión de las dependencias, en relación con la misión y la visión de la UNED que, articulado con el Plan Nacional de la Educación Superior Universitaria Estatal 2011-2015 y el Plan de Desarrollo Institucional PDI 2011-2015, permite una mejor administración de la universidad. 
2. Dar respuesta a la demanda de impresos varios y menores para el buen funcionamiento de las dependencias así como de las prioridades de la institución y sus compromisos (131\%).

3. Promocionar el sello EUNED a través de impresos (177\%).

4. Planificación y programación de la producción (160\%).

5. Promocionar el sello EUNED a través de actividades presenciales de presentación de obras nuevas (100\%).

Otros 4 alcanzaron niveles superiores al $90 \%$ :

1. Producir y reproducir los materiales de matrícula para los diferentes periodos académicos (96\%).

2. Mejoramiento de los procedimientos empleados en las diferentes actividades de la EUNED (95\%).

3. Desarrollar la venta de servicios para la obtención de ingresos mediante el aprovechamiento óptimo de la capacidad de la EUNED en producción de bajo costo y alta calidad (95\%).

4. Promocionar el sello EUNED en medios de comunicación (93\%).

Los objetivos que no llegaron a completarse fueron atribuidos por el Director Ejecutivo de la Editorial a razones ajenas a ésta. En específico, el décimo objetivo refiere a la periodicidad de las revistas de la Universidad. Ésta fue irregular durante el periodo 2005-2010. Publicaciones como Innovaciones Educativas y Repertorio Científico no aparecieron durante un año o más. (Muiños Gual, 2011: 23, 58).

El 26 de octubre de 2012, la UNED publicó en Acontecer, boletín institucional, la ejecución de nueve iniciativas que forman parte del Plan de Mejoramiento Institucional (PMI) -derivado del Proyecto de Mejoramiento de la Educación Superior- mismas que pretenden dinamizar la gestión institucional con el fin de mejorar la educación superior a distancia a lo largo y ancho del territorio nacional. De las nueve iniciativas, aquéllas que se relacionan con el quehacer de PROMADE son:

4. Mejorarlaequidaddeacceso delosestudiantesalosrecursosdeaprendizajedigitales y en Internet.

Con una inversión de US\$600.000,00 se pretende asignar dispositivos tecnológicos a los estudiantes en condiciones de vulnerabilidad socioeconómica. La iniciativa beneficiará entre 2000 y 2500 alumnos y tendrán prioridad aquellos quienes no cuenten con redes de apoyo para poder ausentarse del hogar (mujeres jefas de hogar, población con discapacidad, indígenas). 
Se facilitarán además entre 800 y 1000 dispositivos tecnológicos en los centros de recursos académicos (bibliotecas) de los centros universitarios y en las áreas educativas de los centros penales (sin acceso a Internet pero sí a los materiales multimedia por medio de un dispositivo de almacenamiento).

Los estudiantes que requieran el acceso a Internet se les proporcionará una Datacard de $1 \mathrm{MB}$. Además, aquellos que no tengan posibilidades de acceder a Internet, ni siquiera con la Datacard, se les entregará una llave maya (junto con el dispositivo tecnológico) con todos los recursos digitales para los cursos. Esta misma opción se emplearía con los privados de libertad.

[...]

7. Diversificar y ampliar la producción multimedia digital y en Internet.

Con US\$2.080.000.00, se garantizarán los efectos positivos del lenguaje audiovisual en los procesos cognitivos del estudiante en los procesos de enseñanza aprendizaje, mediante la actualización de los recursos técnicos. [...]

9. Sistema de información para el apoyo a la toma de decisiones yla gestión institucional.

La inversión es de US\$2.599.500.00 y comprende:

Fortalecimiento, mejoramiento y modernización de los sistemas de información institucionales, específicamente los que se relacionan con las áreas de Recursos Humanos, Gestión Académica, Gestión de Estudiantes y Financiero-Contable; con el fin de integrarlos para que satisfagan de manera oportuna y pertinente las necesidades de información y de servicios que requieren los usuarios del área estudiantil, académica, administrativa, así como las autoridades universitarias.

Además, como parte de esta iniciativa se actualizará y mejorará la calidad de los datos ya registrados (bases de datos), se harán modificaciones para incluir nueva información relevante para la toma de decisiones y la gestión. ("Nueve iniciativas...", 2012: s. pág.)

\section{Conclusiones}

[...] las editoriales universitarias [...], y sobre todo las que provienen del Estado tienen una función social que es equilibrar, intervenir entre la hiper-concentración de los capitales privados y multinacionales que concentran la mayor cantidad de autores y títulos, y los autores independientes pequeños que no pueden transcender más allá de la frontera o de la región en la que editan.

Carlos Gazzera, Director de la Editorial de la Universidad Nacional de Villa María (Argentina)

El Programa de Material Didáctico Escrito de la UNED es pionero en generación de contenidos, modelo de gestión, incorporación tecnológica y capacitación de recursos humanos en Costa Rica y Centroamérica. Garantiza 
contenidos académicos de calidad por medio de su planificación y desarrollo por especialistas de distintas disciplinas, los editores académicos.

De 2011 a la fecha ha pasado por un proceso de mejora en la unificación de criterios editoriales, perfiles de puestos, cronómetro en la edición e impresión de publicaciones e investigación, entre otros. Los tópicos que no ha podido concretar obedecen en parte a los tiempos que suele imponer la burocracia institucional de la universidad, característica, por lo demás, generalizada en las instituciones de educación superior del sector público en América Latina. Cuestión disímil a lo que acontece con las editoriales académicas de corte comercial.

Uno de los aspectos más relevantes de este Programa es la implementación de una Metodología para la Presentación de los Contenidos en la Unidad Didáctica Modular. Ésta se entiende como el conjunto de materiales y recursos didácticos con un único propósito: tomar en cuenta el tipo de estudiante. Lo cual supone que las UDM deben fundamentarse en un modelo de comunicación didáctica que considere estos aspectos:

- Tener una estructura clara y explícita.

- Permitir que el estudiante comprenda y haga propias las metas de aprendizaje, facilitándole establecer relaciones con sus experiencias previas, intereses y expectativas.

- Presentar los contenidos con una estructura didáctica de la cual el estudiante pueda apropiarse.

- Partir de núcleos temáticos propios de la realidad profesional, y de ser posible, relacionados con el contexto del estudiante y con el mundo del ejercicio práctico de la profesión, de manera que el estudiante le encuentre sentido al conocimiento y lo proyecte funcionalmente.

- Incorporar actividades de autorregulación, las cuales exigen que el estudiante se apropie con los criterios con los que se juzgará su desempeño, y habilidades de auto-observación y planificación de las propias acciones de estudio.

- Una estructura flexible que posibilite la conformación de un cuerpo de conocimientos teóricos y prácticos que mantengan su vigencia en el tiempo.

Aunque es cierto que hay aspectos del modelo de industrias de contenidos fuertemente arraigados en la labor cotidiana de PROMADE como el conocimiento especializado y la digitalización, es necesario potenciar aquéllos comola inmediatezy desintermediación que pueden catapultar a los productos del Programa en materiales asequibles en diversidad de formatos digitales y análogos, cuando la mediación didáctica así lo solicite. No obstante, valores como el nuevo rol de los actores tendrán serias limitantes en este sistema 
editorial porque la obligatoriedad de calidad y legitimidad constriñe la participación de aquéllos que pueden ser autores, revisores y editores de obras que han de ser punto de partida del proceso de enseñanza-aprendizaje en el modelo educativo de la UNED.

\section{Referencias}

"Capítulo 4. La evolución de la educación superior" (2013), en Cuarto Informe Estado de la Educación 2013, San José de Costa Rica: Programa Estado de la Nación (PEN). Disponible en: http://www.es tadonacion.or.cr/files/biblioteca_virtual/educacion/004/9-Cap-4 .pdf

Casani, Fernando; Rodríguez-Pomeda, Jesús y Sánchez, Flor (2012), "Los nuevos modelos de negocio en la economía creativa:Emociones y redes sociales", en Universia Business Review, primer trimestre, 4869. Disponible en: http://ubr.universia.net/pdfs_web/3303.pdf

Castro, Cosette (2008), Industrias de Contenidos en Latinoamérica, Buenos Aires: CEPAL.

Cole, David (2003), Marketing editorial: la guía, México: Fondo de Cultura Económica.

"Editoriales buscan autores en las redes sociales" (2012), en Culturamas. Revista de información cultural en Internet. Disponible en: http://www.culturamas.es/blog/2012/12/21/editoriales-buscan-au tores-en-las-redes-sociales/

Featherstone, Mike (2002), Cultura de consumo y posmodernismo, Buenos Aires: Amorrortu editores.

Guerra González, Jenny Teresita (2009), Hacia un nuevo fenómeno de masificación en la narrativa latinoamericana: producción, circulación y consumo, tesis de maestría, Estudios Latinoamericanos, México: Facultad de Filosofía y Letras, Universidad Nacional Autónoma de México.

IV Informe Estado de la Región en Desarrollo Humano Sostenible 2011 (2012), San José de Costa Rica: CONARE. Disponible en: http:// www.estadonacion.or.cr/index.php/prensa/carpetas/centroameri $\mathrm{ca}$ /informe-iv

Lobo Solera, Nidia y Fallas Araya, Víctor Hugo (2008), La Benemérita Universidad Estatal a Distancia en la Sociedad del Conocimiento, San José de Costa Rica: EUNED.

McGinty, Stephen (1999), Gatekeepers of Knowledge: Journal Editors in Sciences and Social Sciences, Westport: Bergin \& Garvey.

Modelo Pedagógico de la Universidad Estatal a Distancia. (Aprobado por el Consejo Universitario sesión No. 1714, Artículo IV, inciso 3 del 9 de julio del 2004) (2007), San José de Costa Rica: EUNED. 
Muiños Gual, René (2011), Informe Final de Gestión 2005-2011 de la Dirección Editorial, documento de Control Interno de la Editorial de la Universidad Estatal a Distancia de Costa Rica, San José de Costa Rica.

"Nueve iniciativas fortalecerán la educación superior a distancia" (2012), en Acontecer. Periódico digital de la Universidad Estatal a Distancia de Costa Rica, 26 de octubre de 2012. Disponible en: http:// web.uned.ac.cr/acontecer/index.php/a-diario/educacion/1581-nue ve-iniciativas-fortaleceran-la-educacion-superior-a-distancia.html

Piedras, Ernesto (2008), "Indicadores de Cultura. Economía cultural y economía creativa", en EstePaís, 04 de enero de 2008, 28-29. Disponible en: http://estepais.com/inicio/historicos/205/29_cultura_ indicadores_piedras.pdf

POA (Plan Operativo Anual) (2011), San José de Costa Rica: UNED.

"Presentación del Programa de Material Didáctico Escrito" (2012), Portal de Recursos didácticos de la Universidad Estatal a Distancia de Costa Rica. Disponible en: http://recdidacticos.uned.ac.cr/ promade/

Programa de Apoyo Curricular y Evaluación de los Aprendizajes de la Universidad Estatal a Distancia de Costa Rica (2012). Disponible en: www.uned.ac.cr/pace

Ramírez Ramírez, Celedonio (2006), La Tercera Revolución Educativa Costarricense. Memoria de la creación y puesta en marcha de la UNED (1975-1982), San José de Costa Rica: EUNED.

Rausell Köster, Paul y Carrasco Arroyo, Salvador (2003), "Algunos Apuntes Sobre la Economía de la Comunicación y La Cultura", en Jordan Antuñano (ed.), Política Económica: Fundamentos, Objetivos e Instrumentos, Valencia: Editorial Tirant Lo Blanch.

Román González, Roberto (2011), Plan de Trabajo 2011-2015 de la Dirección de Producción de Materiales Didácticos, San José de Costa Rica: UNED/Dirección de Producción de Materiales Didácticos. Disponible en: http://recdidacticos.uned.ac.cr/recursos/docs/plan_ trabajo.pdf (2012), Informe de Labores de la Dirección de Producción de Materiales Didácticos: Julio 2011 a Julio 2012 y Propuestas de Acciones hasta el 2015, San José de Costa Rica: UNED/Vicerrectoría Académica/Dirección de Producción de Materiales Didácticos. Disponible en: http://recdidacticos.uned.ac.cr/recursos/docs/informe dpmd_2011-2012.pdf

Tejeda Gómez, María Alejandra (2009), “Adelantos y retos en la medición de la información y el impacto de las publicaciones científicas en el mundo académico", en Memorias del VII Congreso InternacionaldePublicaciones Universitarias delberoamérica.Conocimiento y Sociedad, Bogotá: ASEUC/Cámara Colombiana del Libro/La Librería de la U. 
Wolford, Josh (2012), "Twitter Fiction Festival Selects Participants, Starts Tomorrow", en WebProNews, 27 de noviembre de 2012. Disponibleen:http://www.webpronews.com/twitter-fiction-festivalselects-participants-starts-tomorrow-2012-11

Para citar este artículo como revista electrónica:

Guerra González, Jenny Teresita. 2016. “La producción editorial didáctica de la Universidad Estatal a Distancia de Costa Rica: un diagnóstico a partir del modelo de industrias de contenidos". InvestigaciónBibliotecológica:Archivonomía,Bibliotecologíaelnformación.68:123-151.Aquíseagregaladirecciónelectrónica(Consultado el día-mes-año)

Para citar este artículo tomado de un servicio de información:

Guerra González, Jenny Teresita. 2016. “La producción editorial didáctica de la Universidad Estatal a Distancia de Costa Rica: un diagnóstico a partir del modelo de industrias de contenidos". InvestigaciónBibliotecológica:Archivonomía,Bibliotecologíaelnformación.68:123-151.En:Aquíseagregaelnombredelserviciodeinformación y la dirección electrónica (Consultado el día-mes-año) 
